\title{
Apelin signaling antagonizes Ang II effects in mouse models of atherosclerosis
}

\author{
Hyung J. Chun, ${ }^{1}$ Ziad A. Ali, ${ }^{1}$ Yoko Kojima, ${ }^{1}$ Ramendra K. Kundu, ${ }^{1}$ Ahmad Y. Sheikh, ${ }^{1}$ Rani Agrawal, ${ }^{2}$ \\ Lixin Zheng, ${ }^{3}$ Nicholas J. Leeper, ${ }^{1}$ Nathan E. Pearl, ${ }^{2}$ Andrew J. Patterson, ${ }^{2}$ Joshua P. Anderson, ${ }^{1}$ \\ Philip S. Tsao, ${ }^{1}$ Michael J. Lenardo, ${ }^{3}$ Euan A. Ashley, ${ }^{1}$ and Thomas Quertermous ${ }^{1}$
}

${ }^{1}$ Department of Medicine and 2Department of Anesthesiology, Stanford University School of Medicine, Stanford, California, USA. ${ }^{3}$ Laboratory of Immunology, National Institute of Allergy and Infectious Diseases, NIH, Bethesda, Maryland, USA.

\begin{abstract}
Apelin and its cognate G protein-coupled receptor APJ constitute a signaling pathway with a positive inotropic effect on cardiac function and a vasodepressor function in the systemic circulation. The apelin-APJ pathway appears to have opposing physiological roles to the renin-angiotensin system. Here we investigated whether the apelin-APJ pathway can directly antagonize vascular disease-related Ang II actions. In ApoE-KO mice, exogenous Ang II induced atherosclerosis and abdominal aortic aneurysm formation; we found that coinfusion of apelin abrogated these effects. Similarly, apelin treatment rescued Ang II-mediated increases in neointimal formation and vascular remodeling in a vein graft model. NO has previously been implicated in the vasodepressor function of apelin; we found that apelin treatment increased NO bioavailability in ApoE-KO mice. Furthermore, infusion of an NO synthase inhibitor blocked the apelin-mediated decrease in atherosclerosis and aneurysm formation. In rat primary aortic smooth muscle cells, apelin inhibited Ang II-mediated transcriptional regulation of multiple targets as measured by reporter assays. In addition, we demonstrated by coimmunoprecipitation and fluorescence resonance energy transfer analysis that the Ang II and apelin receptors interacted physically. Taken together, these findings indicate that apelin signaling can block Ang II actions in vascular disease by increasing NO production and inhibiting Ang II cellular signaling.
\end{abstract}

\section{Introduction}

Recent studies have suggested that the apelin-APJ pathway is a potent regulator of cardiovascular function. The apelin ligand is translated as a 77-amino acid prepropeptide and cleaved to shorter peptides that bind the APJ GPCR $(1,2)$. Apelin is expressed primarily in the endothelium and acts both locally and via endocrine signaling to activate APJ, which is expressed on myocardial cells, endothelial cells, and some SMCs (3-6). In isolated rat cardiomyocytes and intact hearts as well as with in vivo rat and mouse models, apelin has been shown to have a positive effect on contractility (7-10). Experiments employing myocardial injury models have suggested that apelin also has a positive inotropic effect on failing myocardium $(8,11)$. In the vasculature, studies support a vasodepressor role in both the arterial and venous circulation and suggest that these effects are mediated at least in part by NO (12-15).

The apelin-APJ pathway has opposing actions to the renin-angiotensin system in a number of physiologic and pathophysiologic settings. While Ang II increases vascular tone and raises blood pressure, apelin is a vasodilator and lowers blood pressure; Ang II and apelin are expressed in functionally opposing neurons in the hypothalamus and Ang II promotes central arginine vasopressin release, while apelin inhibits its release; glucocorticoids increase Ang II levels and suppress apelin expression; in heart failure, Ang II levels rise, whereas apelin levels fall $(3,12-18)$. In addition, there is some evidence for direct counterregulation of the renin-angioten-

Nonstandard abbreviations used: AAA, abdominal aortic aneurysm; AT1R, Ang II receptor type I; CFP, cyan fluorescent protein; EPR, electron paramagnetic resonance; FRET, fluorescence resonance energy transfer; L-NAME, L-N ${ }^{\mathrm{G}}$-nitroarginine methyl ester; RASMC, rat aortic SMC; SRE, serum response element; YFP, yellow fluorescent protein.

Conflict of interest: The authors have declared that no conflict of interest exists. Citation for this article: J. Clin. Invest. 118:3343-3354 (2008). doi:10.1172/JCI34871. sin system by apelin-APJ. Ishida et al. have reported differences in blood pressure response in mice lacking both the Ang II receptor type I (AT1R) and APJ compared with mice lacking only AT1R (13). In a hypertensive model of heart failure, Iwanaga et al. showed that Ang II inhibits expression of apelin and APJ, perhaps contributing to cardiac decompensation (19).

In studies reported here we have investigated the hypothesis that apelin can antagonize the vascular disease-promoting actions of Ang II. In the ApoE-deficient atherosclerosis model (ApoE-KO), apelin was shown to mitigate the Ang II-mediated increase in atherosclerosis burden and to inhibit abdominal aortic aneurysm (AAA) formation and rupture. In a vein graft model, apelin inhibited the Ang II-mediated increase in neointimal formation. In both of these in vivo models, apelin was shown to significantly decrease vascular $\mathrm{O}_{2}{ }^{-}$. Finally, our in vitro studies demonstrate that the apelin-APJ pathway directly antagonizes Ang II signaling to the nucleus through cellular signaling crosstalk.

\section{Results}

Apelin inhibits native and Ang II-accelerated atherosclerosis in murine models. We first conducted experiments to investigate the effect of endogenous apelin signaling on atherosclerosis. Apelin-ApoE double-knockout mice were generated, and atherosclerotic burden in these animals was compared with that in $A p o E$-null mice (6). In the thoracoabdominal aorta, apelin- $A p o E$ double knockouts developed significantly greater atherosclerotic lesion area compared with that in ApoE-KO mice, irrespective of the type of diet that was administered (2.53-fold increase, $P<0.01$ for normal chow; 1.50 -fold increase, $P<0.01$ for Western diet) (Figure 1A).

To test whether the antiatherosclerotic effect of apelin might be due in part to inhibition of Ang II disease processes, we used 2 models of exogenous Ang II-exacerbated vascular disease in ApoE- 


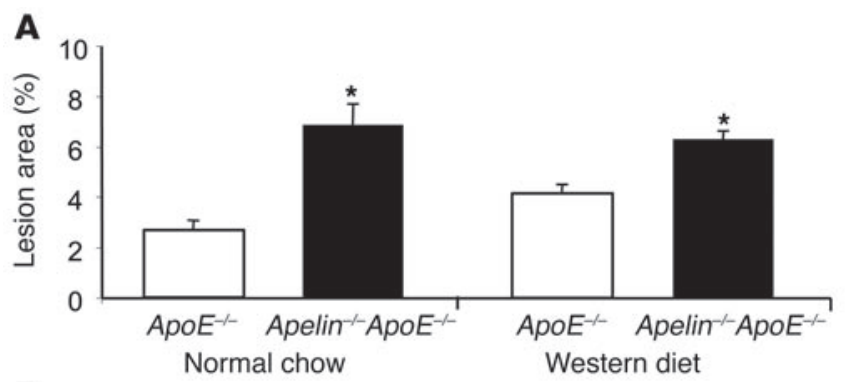

B

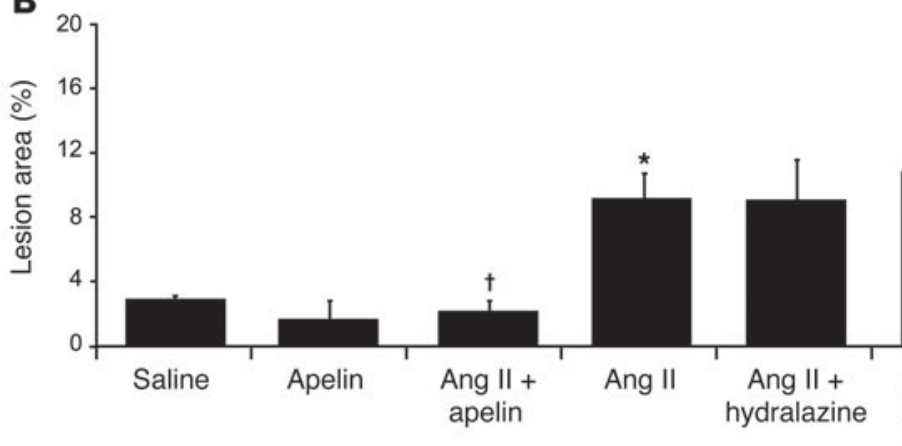

C

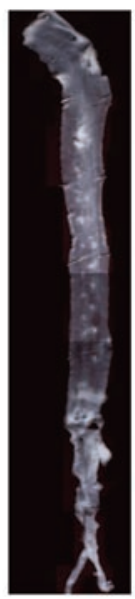

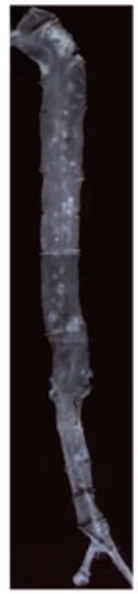

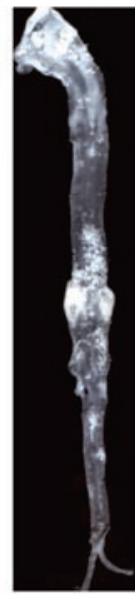

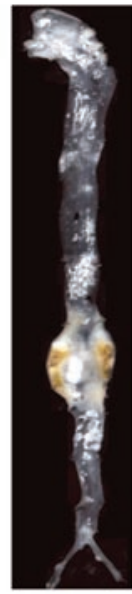

\section{Figure 1}

Apelin inhibits atherosclerosis in the ApoE-null model. (A) Apelin-ApoE double-knockout and $A p o E-K O$ mice were fed normal chow or high-fat Western diet, and atherosclerosis burden was evaluated by en face analysis of the thoracoabdominal aorta. Apelin-ApoE double-knockout mice developed significantly greater atherosclerotic lesions compared with $A p o E-K O$ mice on both diets $\left(2.53\right.$-fold increase, ${ }^{*} P<0.01$ for normal chow; 1.50 -fold increase, ${ }^{*} P<0.01$ for Western diet). (B) Coinfusion of apelin mitigated the increased atherosclerotic lesion burden caused by Ang II when coadministered by osmotic minipump $\left({ }^{*} P<0.01\right.$, Ang II compared with saline; ${ }^{\dagger} P<0.001$, Ang II plus apelin compared with Ang II). Animals receiving Ang II and hydralazine had an atherosclerosis burden similar to that seen in the Ang II group. Administration of the NOS inhibitor L-NAME mitigated the beneficial effects of apelin, bringing atherosclerotic lesion burden to similar levels as mice infused with Ang II alone. (C) ApoE-KO mice treated with Ang II for 4 weeks showed a significant increase in aneurysm formation, and this effect was mitigated by apelin treatment. Administration of hydralazine with Ang II did not protect ApoE-KO mice from aneurysm formation. Mice treated with Ang II plus apelin plus L-NAME demonstrated aneurysm formation similar to Ang II mice. Representative aortas are shown for each experimental group. Original magnification, $\times 5$.
KO mice (Supplemental Figure 1; supplemental material available online with this article; doi:10.1172/JCI34871DS1). We employed 4 treatment groups for the initial study: (a) saline, (b) apelin, (c) Ang II, and (d) Ang II plus apelin. These groups demonstrated comparable lipid profiles and body weights (Supplemental Table 1 ). In this model, extent of aortic atherosclerosis and AAA formation and rupture were evaluated (20-23). In the thoracoabdominal aorta, ApoE-KO mice treated with Ang II developed significantly greater atherosclerotic area compared with that in saline controls (3.4-fold increase, $P<0.01$, Ang II versus saline), but concurrent infusion of apelin almost totally blocked this increase $(P<0.001$, Ang II plus apelin versus Ang II) (Figure 1, B and C). In the aortic root, there was a reduction in atherosclerotic burden in apelintreated mice compared with saline-treated controls $(P<0.05)$, but neither Ang II treatment or cotreatment with apelin and Ang II resulted in any significant difference (Supplemental Figure 2).

To investigate the mechanisms underlying these apelin-mediated effects, we evaluated aortic atherosclerosis burden of additional experimental groups with the en face assay. To exclude the potentially confounding effect of blood pressure reduction

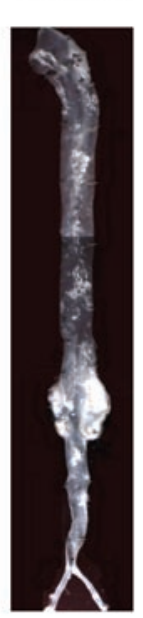

by apelin in the Ang II plus apelin group compared with Ang IItreated animals, we examined the degree of atherosclerosis in animals coadministered Ang II and hydralazine. Mice in the Ang II plus apelin group demonstrated a lower systolic blood pressure compared with that in the Ang II group $(114 \pm 8 \mathrm{mmHg}$ versus $151 \pm 7 \mathrm{mmHg}, P<0.01$ ), and mice administered Ang II with hydralazine in their drinking water had blood pressure readings similar to those in the Ang II plus apelin group $(115 \pm 10 \mathrm{mmHg}$ versus $114 \pm 8 \mathrm{mmHg}, P=0.99)$ (Figure 2 ). However, the severity of atherosclerosis remained comparable to the Ang II group despite the reduction in blood pressure in the Ang II plus hydralazine group (Figure 1, B and C). These findings confirm that the beneficial effects of apelin on atherosclerosis are not mediated by a reduction in blood pressure.

As previous studies have cited a role for $\mathrm{NO}$ as a potential mediator of the beneficial effects of apelin, in a separate group of animals we concurrently administered the NOS inhibitor $\mathrm{L}-\mathrm{N}^{\mathrm{G}}$-nitroarginine methyl ester (L-NAME) with apelin and Ang II to determine if the disease-ameliorating effect of apelin might be due to increased NO bioavailability. Administration of L-NAME completely abrogat- 


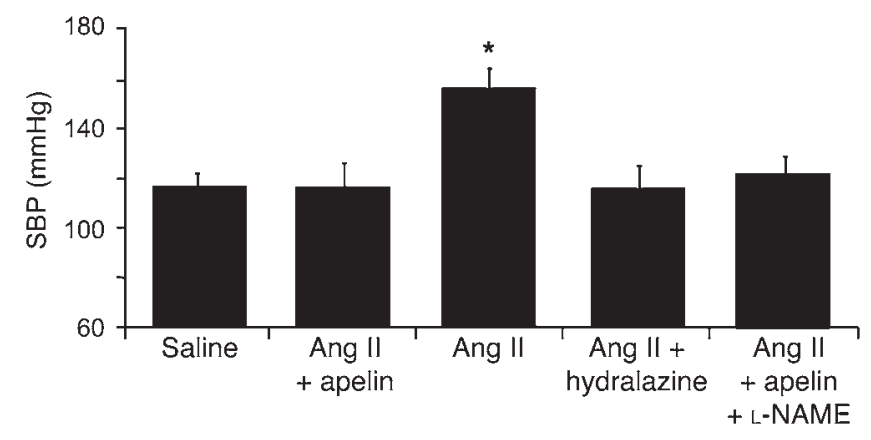

Figure 2

Apelin inhibits the Ang II-mediated increase in blood pressure. Systolic blood pressure (SBP) in the Ang II group was significantly increased compared with the saline control group $(151 \pm 7 \mathrm{mmHg}$ versus $113 \pm 4 \mathrm{mmHg}$; $\left.{ }^{*} P<0.01\right)$. Concurrent infusion of apelin or administration of oral hydralazine eliminated this increase in blood pressure. Addition of L-NAME did not significantly increase the blood pressure.

ed the apelin-mediated reduction in atherosclerosis, and these mice developed atherosclerosis comparable to the Ang II alone group $(10.8 \% \pm 2 \%$ versus $9.2 \% \pm 4.6 \%, P=0.52)$ (Figure $1, \mathrm{~B}$ and $\mathrm{C}$ ).

We next investigated AAA formation in the same cohort of mice. We did not observe AAA formation in the saline or apelin groups, whereas the majority of mice (89\%, 16 of 18 mice) infused with Ang II developed AAA or dissection within 4 weeks (Table 1 and Figure 1C). Coinfusion of apelin with Ang II dramatically attenuated this effect, significantly reducing the incidence of AAA formation $(5 \%, 1$ of $20 ; P<0.001)$. Also, the addition of apelin to Ang IItreated animals reduced sudden death due to aortic rupture compared with Ang II-treated mice (0 of 20 versus 7 of 18 mice; $P<0.05$ ) (Table 1). Reduction of blood pressure with hydralazine did not afford protection against AAA formation or sudden death in the Ang II group. Moreover, in the Ang II plus apelin plus L-NAME group, the protective benefit of apelin on AAA formation ( $80 \%, 8$ of 10 mice) and death due to aortic rupture $(30 \%, 3$ of 10 mice) was abrogated (Table 1 ). These findings suggest that the protective benefits of apelin in our in vivo model systems are at least in part mediated by NO.

To further evaluate the ability of apelin to block vascular disease processes mediated by Ang II, we employed a vein graft model in the ApoE-KO background characterized by acute endothelial loss and neointimal hyperplasia (24). Mean neointimal and vessel wall areas were significantly reduced in the apelin group compared with the saline group (77\% reduction, $P<0.001$; $42 \%$ reduction, $P<0.05$, respectively) (Figure $3 \mathrm{~A})$. The Ang II group demonstrated increased vein graft neointimal and wall areas compared with the saline group (95\% increase, $P<0.05 ; 33 \%$ increase, $P<0.05$, respectively). However, coinfusion of apelin with Ang II rescued this phenotype, with significant decreases in both neointimal area $(71 \%$ decrease, $P<0.001)$ and mean vessel wall area $(34 \%$ decrease, $P<0.05)$ (Figure $3 \mathrm{~A})$. Neointima/vessel area ratios demonstrate that the beneficial effect of apelin treatment is more pronounced on neointimal hyperplasia $(P<0.05$, apelin compared with saline; $P<0.05$, Ang II plus apelin compared with Ang II). As neointimal hyperplasia is mediated largely through migration and proliferation of SMCs, we quantified SMC $\alpha$-actin immunohistochemistry on sections. Apelin infusion significantly decreased the SMC component, both in total (47\% reduction, $P<0.001$ ) and when defined as a proportion of the total vessel wall $(0.27 \pm 0.05$ versus $0.47 \pm 0.08$, respectively; $P<0.05$ ), compared with saline-treated controls (Figure 3B). Infusion of Ang II increased SMC content (40\% increase versus saline control, $P<0.01$ ), and infusion of apelin in Ang II-treated animals reduced SMC content (65\% reduction, $P<0.001$ ) and proportion $(0.21 \pm 0.03$ versus $0.48 \pm 0.04 ; P<0.001)$ compared with animals treated with Ang II infusion alone (Figure 3, B and C).

Apelin regulates production of $\mathrm{NO}$ and reactive oxygen species in the vessel wall. To further address the mechanism of apelin's effects in these models, we investigated the production of NO. NO has been implicated in apelin-mediated vasodepressor function and is well known to have salutary vascular actions. Net NO bioavailability, measured using $\mathrm{Fe}(\mathrm{DETC})_{2}$ electron paramagnetic resonance (EPR) spectroscopy, was significantly increased by approximately 2 -fold in apelin-treated mice compared with saline-treated controls $(P<0.001)$ (Figure 4A). Addition of the NOS inhibitor L-NAME significantly reduced net NO bioavailability to levels similar to saline-treated mice $(P<0.05)$, further suggesting that apelin stimulated NOS activity. Although there was no significant difference in net NO bioavailability in the Ang II plus apelin group compared with the Ang II group, addition of L-NAME in the Ang II plus apelin group reduced NO bioavailability to basal levels $(P<0.05)$, suggesting that apelin induces NO synthesis despite high levels of constitutive oxidative stress induced by Ang II in ApoE-KO mice.

Numerous proatherosclerotic effects of Ang II have been linked to $\mathrm{O}_{2}{ }^{\circ-}$ signaling in vascular tissue, thus we measured $\mathrm{O}_{2}{ }^{--}$production in the diseased vessel wall of the ApoE-KO animals. Total vascular $\mathrm{O}_{2}{ }^{-}$production, measured by lucigeninenhanced chemiluminescence, was approximately $50 \%$ lower in apelin-treated aortas compared with saline controls $(P<0.01)$ (Figure 4B). Ang II infusion stimulated a 10 -fold increase in total chemiluminescence $(P<0.001)$, which was significantly

\section{Table 1}

AAA formation and death due to aneurysm rupture in ApoE-KO mice

\begin{tabular}{|c|c|c|c|c|c|c|}
\hline & Saline & Apelin & Ang II plus apelin & Ang II & Ang II plus hydralazine & $\begin{array}{l}\text { Ang II plus apelin } \\
\text { plus L-NAME }\end{array}$ \\
\hline Aneurysm or dissection & $0 / 15(0 \%)$ & $0 / 18(0 \%)$ & $1 / 20(5 \%)$ & $16 / 18(89 \%)$ & $8 / 11(73 \%)$ & $8 / 10(80 \%)$ \\
\hline Death & $0 / 15(0 \%)$ & $0 / 18(0 \%)$ & $0 / 20(0 \%)$ & $7 / 18(39 \%)$ & $3 / 11(27 \%)$ & $3 / 10(30 \%)$ \\
\hline
\end{tabular}

ApoE-KO animals treated with Ang II for 4 weeks showed a significant increase in aneurysm formation, and this effect was mitigated by apelin treatment (16 of 18 compared with 1 of 20 mice; $P<0.001$ ). Addition of apelin reduced sudden death due to AAA rupture compared with mice treated with only Ang II ( 0 of 20 versus 7 of 18 mice; $P<0.05$ ). In the Ang II plus apelin plus L-NAME group, the protective benefit of apelin on AAA formation and death due to aortic rupture was abrogated (analysis by Fisher's exact test). Data are shown as number affected/total (\%). 
A

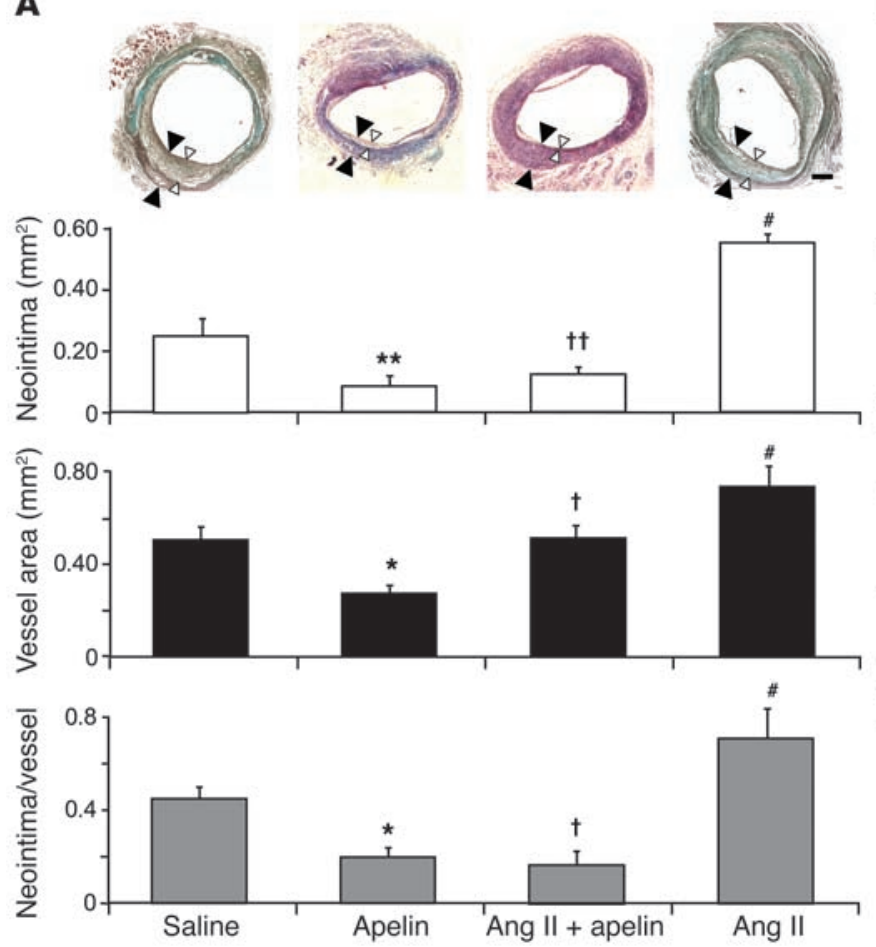

B

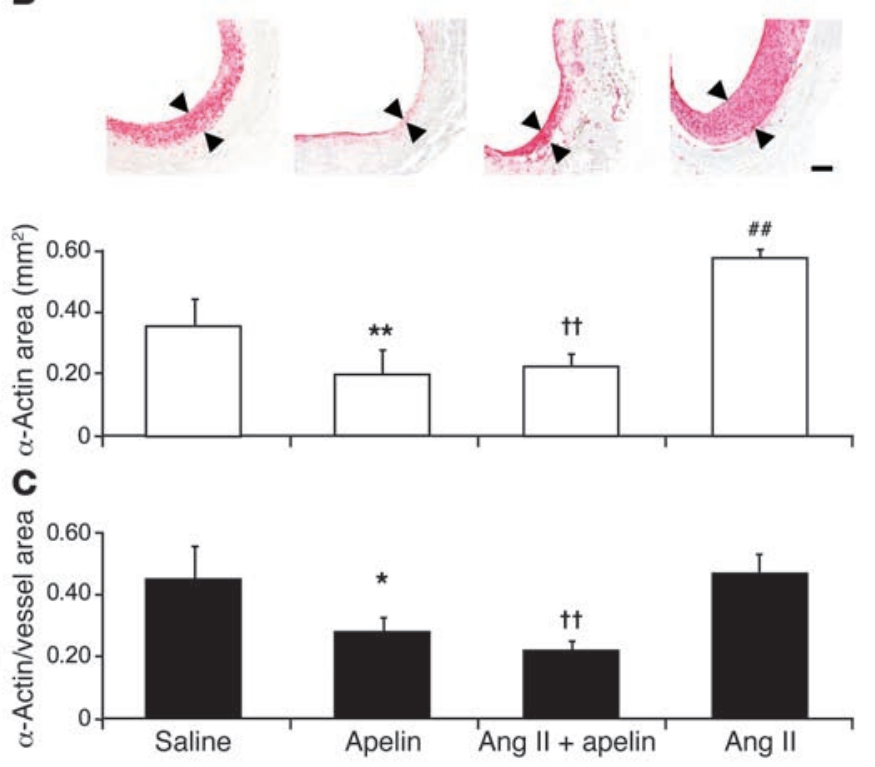

Figure 3

Apelin inhibits Ang II-mediated progression of atherosclerosis in a vein graft model. (A) Top row shows representative images of the vein graft cross sections stained with Masson/Goldner. Neointimal area, total vessel wall area, and neointimal vessel area were determined and compared among the experimental groups indicated. Apelin infusion significantly decreased each of these parameters compared with saline control group $\left({ }^{\star} P<0.05,{ }^{\star \star} P<0.001\right)$. Ang II infusion significantly increased all parameters in comparison to the control group $\left({ }^{\#} P<0.05\right)$, and this effect was significantly inhibited when apelin was administered along with Ang II $\left({ }^{\dagger} P<0.05,{ }^{\dagger} P<<0.001\right)$. Neointima/vessel area ratios demonstrate that the beneficial effect of apelin treatment is more pronounced on neointimal hyperplasia. White arrows denote neointima and black arrows denote total vessel wall thickness. Scale bar: $100 \mu \mathrm{m}$. (B) SMC contribution to vascular wall disease was assessed by measuring the area occupied by SMC $\alpha$-actin-stained cells in serial sections. Micrographs show representative images of the vein graft cross sections stained with $\alpha$-actin antibody. Infusion of Ang II increased SMC content (\#\# $P<0.01)$. Apelin infusion decreased the measured SMC component of the vessel cross-sectional area $\left({ }^{* *} P<0.001\right)$ compared with saline-treated animals and also decreased the SMC area when combined with Ang II infusion compared with Ang II infusion alone $\left({ }^{\dagger+} P<0.001\right)$. Black arrows denote $\alpha$-actin-positive staining. Scale bar: $100 \mu \mathrm{m}$. (C) The contribution of SMCs was also assessed as a proportion of the total vessel wall area. Apelin infusion decreased the proportion of SMC $\left({ }^{*} P<0.05\right)$ compared with saline-treated animals and also decreased the SMC proportion when combined with Ang II infusion $\left({ }^{\dagger+} P<0.001\right)$ compared with Ang II infusion alone.

decreased by apelin $(P<0.01)$. When aortas from saline, Ang II, or Ang II plus apelin-treated animals were incubated with L-NAME, total $\mathrm{O}_{2}{ }^{-}-$levels were not significantly different, suggesting that the endothelial contribution to $\mathrm{O}_{2}{ }^{--}$was minimal. However, incubation of aortic tissue from apelin-treated mice with L-NAME led to a 2 -fold increase in total $\mathrm{O}_{2}{ }^{-}$, indicating that under basal conditions the endothelium in apelin-treated mice was producing $\mathrm{NO}$ capable of scavenging $\mathrm{O}_{2}{ }^{-}$.

To confirm the chemiluminescence findings and investigate the cellular source of $\mathrm{O}_{2}^{-}{ }^{-}$in the aorta, we measured its production using dihydroethidium oxidative fluorescence microtopography and conducted comparison among the different layers of the vessel wall. The endothelial layer contributed only a small amount of fluorescence, and there were not significant differences between $\mathrm{O}_{2}{ }^{--}$production levels among the different experimental conditions (Figure 4C). Apelin dramatically decreased $\mathrm{O}_{2}{ }^{-}$production, compared with the saline control group, for both the medial and adventitial vessel layers (78\% and $88 \%$ reduction, respectively; $P<0.001$ for both) (Figure 4C). Apelin also significantly decreased the $\mathrm{O}_{2}{ }^{-}$production in the media and adventitia when combined with Ang II compared with Ang II infusion animals, although to a lesser degree (53\% and $45 \%$, reduction, respectively; $P<0.01$ for both). A similar study was also performed using the vein graft model described above, and $\mathrm{O}_{2}{ }^{--}$production was evaluated in the endothelial and neointimal layers. There was no difference in $\mathrm{O}_{2}{ }^{-}$ production in the endothelium among the different conditions (Supplemental Figure 3). $\mathrm{O}_{2}{ }^{--}$production was similarly found to be decreased in the neointima in mice treated with apelin $(54 \%$ reduction, $P<0.05$, apelin versus saline; $48 \%$ reduction, $P<0.05$, Ang II plus apelin versus Ang II alone).

Apelin regulates Ang II-mediated nuclear signaling. To investigate the cellular mechanism by which apelin inhibits the atherosclerosis-promoting actions of Ang II, we evaluated the effect of apelin on Ang IImediated nuclear signaling. We first evaluated the phosphorylation of ERK1/2. When primary rat aortic SMCs (RASMCs), which express both AT1R and APJ, were treated with apelin and Ang II, the degree of ERK phosphorylation was significantly decreased compared with that during stimulation with Ang II alone (5 minutes, $P<0.01 ; 15$ minutes, $P<0.05$ ) (Figure 5A). We next assessed known transcriptional targets of Ang II signaling using luciferase 


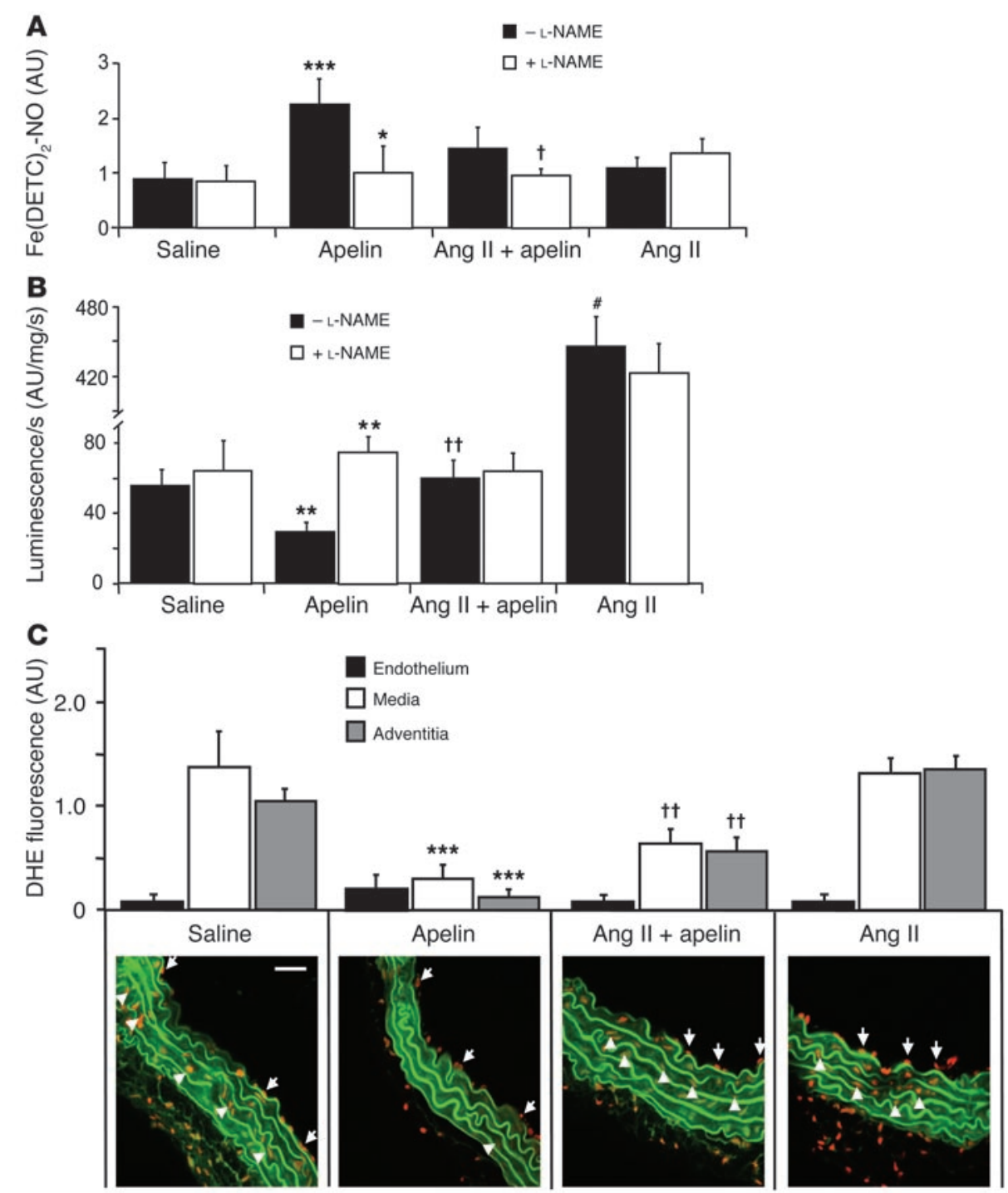

Figure 4

Apelin increases vascular wall NO bioavailability and suppresses disease-related $\mathrm{O}_{2}{ }^{--}$production. (A) EPR spectroscopy was employed to measure $\mathrm{NO}$ in lung homogenates of $A p o E-\mathrm{KO}$ mice. Apelin infusion increased net lung $\mathrm{NO}$ levels compared with saline-treated controls $\left({ }^{\star \star \star} P<0.001\right)$. Inhibition of NOS by L-NAME attenuated net NO levels in apelin $\left({ }^{\star} P<0.05\right)$ and Ang II plus apelin $\left({ }^{\dagger} P<0.05\right)$ lung homogenates, suggesting apelin stimulates NOS activity. (B) Aortic $\mathrm{O}_{2}{ }^{-}$- production, measured by lucigenin, was attenuated in apelin-treated mice $\left({ }^{* *} P<0.01\right)$ and increased in Ang II-treated mice ( $\left.{ }^{P}<0.001\right)$ compared with saline-treated controls. Incubation with L-NAME increased luminescence in apelin-infused mice ( ${ }^{\star \star} P<0.01$, intra-group comparison), suggesting apelin stimulated $\mathrm{NO}$ was quenching $\mathrm{O}_{2}{ }^{*-}$ produced in the vessel wall. Luminescence was reduced in apelin-treated mice, even when coadministered with Ang II compared with animals treated with Ang II alone $\left({ }^{\dagger \dagger} P<0.01\right)$. (C) Dihydroethidium (DHE) fluorescence microscopy allowed topographic assessment of $\mathrm{O}_{2}{ }^{*-}$ production in the vessel wall. There was no significant difference in endothelial $\mathrm{O}_{2}{ }^{--}$production among the different experimental groups. Apelin decreased $\mathrm{O}_{2}{ }^{-}$production compared with the saline control group for both the medial and adventitial vessel layers $\left(78 \%,{ }^{* * *} P<0.001 ; 88 \%\right.$, ${ }^{* * *} P<0.001$, respectively). Apelin also decreased $\mathrm{O}_{2}{ }^{-}$- production in the media and adventitia when combined with Ang II, compared with the Ang II alone animals, although to a lesser degree $\left(53 \%,{ }^{\dagger} P<0.01 ; 45 \%,{ }^{\dagger \dagger} P<0.01\right.$, respectively). Aortic elastic laminae exhibit green autofluorescence. White arrows denote endothelial $\mathrm{O}_{2}{ }^{*-}$ producing cells, and white arrowheads denote medial $\mathrm{O}_{2}{ }^{-}-$producing cells. Scale bar: $5 \mu \mathrm{m}$.

reporter constructs with NF-кB, nuclear factor of activated T cells (NFAT), activator protein-1 (AP-1), and serum response element (SRE) consensus response sequences. All of these nuclear pathways have been described to be downstream targets of Ang II signaling (25-27). HEK293 cells, which express low levels of endogenous APJ (our unpublished observations), were cotransfected with an AT1R expression construct and the respective luciferase reporter plasmid and were stimulated with Ang II or Ang II plus apelin (Figure
5B). Ang II produced a significant increase in transcription of each reporter, and this increase was abrogated when cells were cotreated with apelin. To determine the specificity of apelin's inhibitory effects on Ang II nuclear signaling, we measured the effects of apelin on TNF- $\alpha$ - and $\mathrm{H}_{2} \mathrm{O}_{2}$-induced NF- $\kappa \mathrm{B}$ transcriptional activity (Figure $5 \mathrm{C})$. In neither case did apelin significantly diminish the reporter activity of the NF- $\mathrm{KB}$ reporter construct. To exclude the possibility of apelin competitively binding to AT1R, we performed ligand 
A
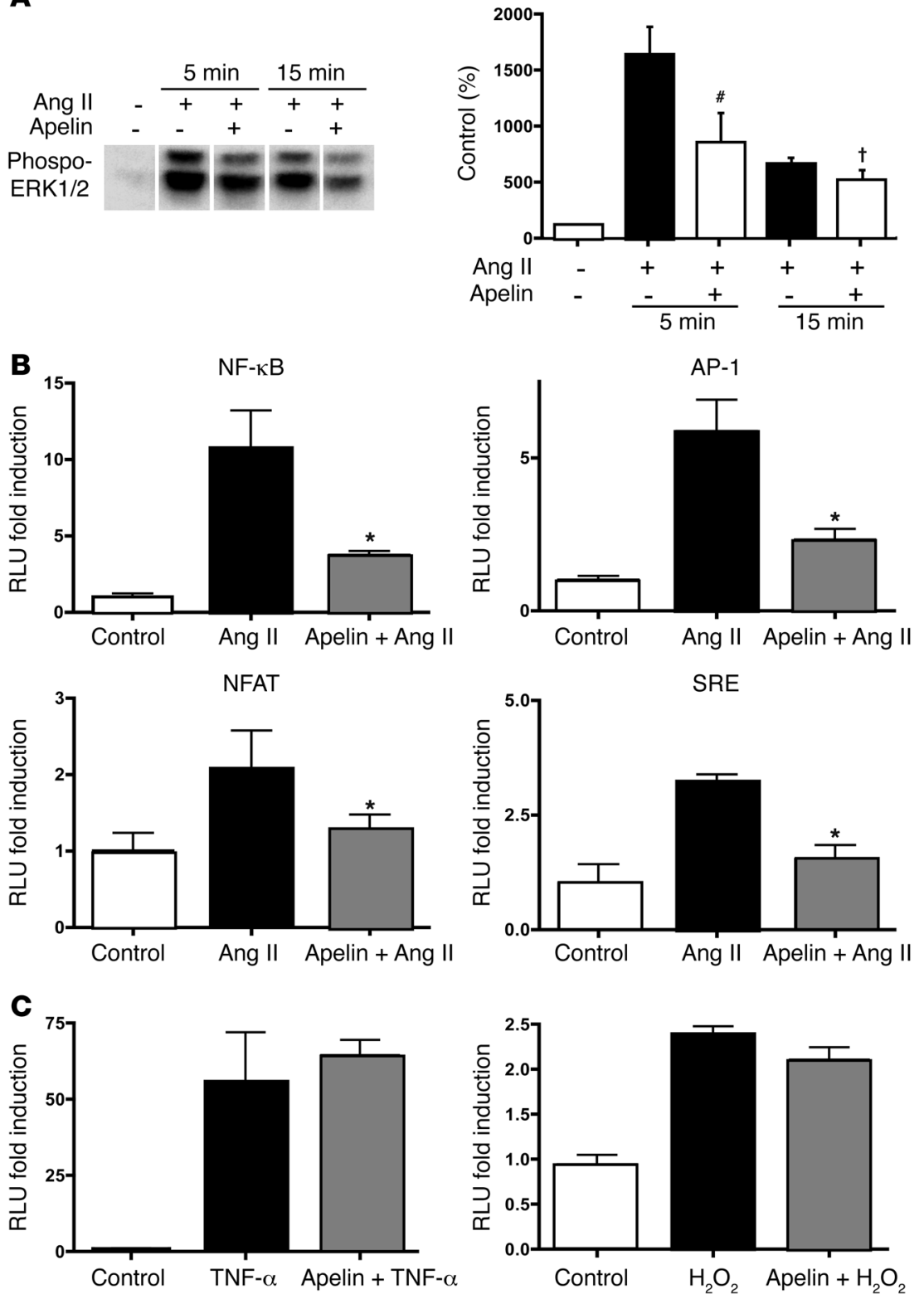

Figure 5

Apelin inhibits Ang II cellular signaling. (A) Ang II stimulation of RASMCs results in phosphorylation of ERK1/2, with marked inhibition by cotreatment with apelin at both 5 minutes $\left({ }^{\sharp} P<0.01\right)$ and 15 minutes $\left({ }^{\dagger} P<0.05\right)$. The lanes are from the same gel and were noncontiguous. (B) Luciferase reporter constructs containing DNA binding sequences for NF- $\mathrm{KB}$, nuclear factor of activated T cells (NFAT), activator protein-1 (AP-1), and SRE were transfected into HEK293 cells and assayed for increased transcription in response to Ang II, in the absence and presence of apelin. All 4 reporters showed decreased activation when cells were cotreated with apelin and Ang II ( $\left.{ }^{\star} P<0.05\right)$. (C) NF-KB reporter activity stimulated by TNF- $\alpha$ and $\mathrm{H}_{2} \mathrm{O}_{2}$ was not affected by apelin treatment $(P=0.97$ and $P=0.18$, respectively).

Ang II and apelin receptors interact in vitro and in vivo. Previous studies have shown GPCR heterodimerization as a mechanism for modulating the function of this class of receptors, and specifically, AT1R has been shown to interact with the bradykinin receptor and the Ang II type 2 receptor in a functional manner (28-30). To assess possible receptor heterodimerization between APJ and AT1R, we transfected HEK293 cells with expression constructs for AT1R tagged with GFP and APJ tagged with HA. We first confirmed that the AT1R-GFP fusion protein was functional by demonstrating induction of the NF-кB luciferase reporter when stimulated with Ang II (data not shown). Immunoprecipitation using $\alpha$-GFP antibody and immunoblotting with $\alpha$-HA antibody demonstrated specific interaction between recombinant APJ and AT1R proteins (Figure 6A).

As a second, confirmatory methodology for establishing heterodimerization, fluorescence resonance energy transfer (FRET) analysis was performed. HEK293 cells were cotransfected with APJ and AT1R expression constructs, with coding regions fused to either cyan fluorescent protein (CFP) or yellow fluorescent protein (YFP). FRET analysis demonstrated specific interaction between APJ and AT1R but not between either of these receptors and the Fas receptor (Figure 6B).

To determine whether the ligands for the 2 receptors can modulate receptor heterodimerization, we performed coimmunoprecipitation and FRET analyses in the presence of apelin and Ang II. When cells were treated with Ang II, there was a dramatic increase in coimmunoprecipitation of the receptors by 6 hours (Figure 6C). However, this association was only minimally increased by the addition of apelin. FRET association analysis confirmed the increased receptor heterodimerization by Ang II stimulation (Figure 6D). Apelin had a minimal effect on the receptor heterodimerization either alone or in combination with Ang II.

APJ inbibits Ang II signaling independent of apelin. Finally, we investigated the effect of the APJ receptor on Ang II signaling, independent of the apelin ligand. We evaluated ERK1/2 phosphorylation in cells expressing the AT1R alone or AT1R and APJ. ERK1/2 protein phosphorylation was dramatically reduced at both 5 and

binding experiments in AT1R-transfected HEK293 cells with mono5-(and-6)-carboxyfluorescein-labeled (FAM-labeled) Ang II. While a 10-fold excess of unlabeled Ang II eliminated FAM-Ang II binding, unlabeled apelin showed no inhibition even at a 100-fold excess (Supplemental Figure 4). This finding confirmed that the ability of apelin to specifically inhibit Ang II signaling was not mediated through AT1R but through a separate receptor, presumably APJ.
15 minute time points in the presence of APJ (Figure 7A). NF-кB and SRE luciferase reporter activity was measured in cells transfected with an AT1R construct alone or an AT1R construct plus varying amounts of APJ expression construct. Robust stimulation of luciferase activity was noted with Ang II in AT1R-expressing cells, but Ang II signaling was diminished in direct proportion to the level of transfected APJ (Figure 7B). This APJ inhibition appeared specific to 
A

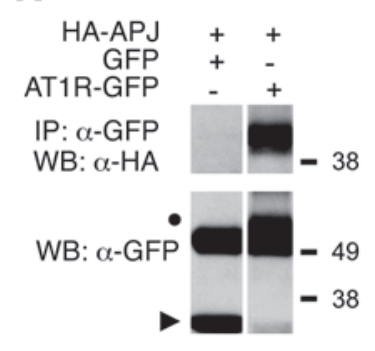

C

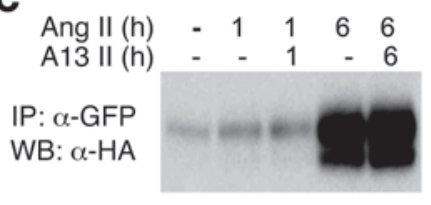

B

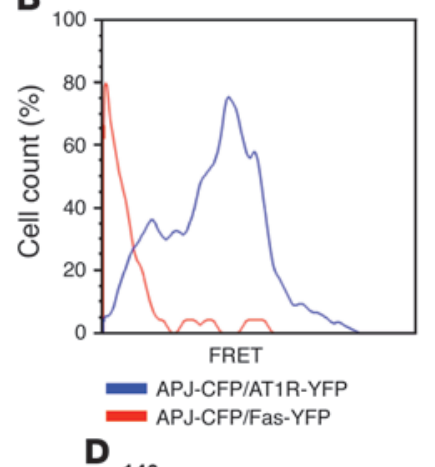

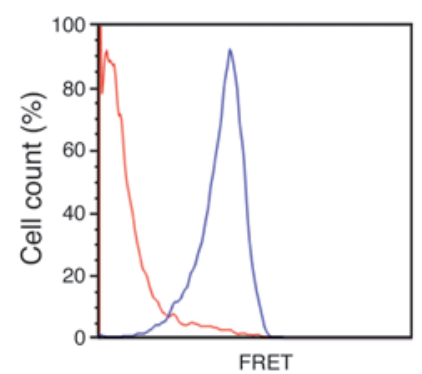

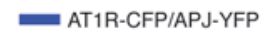
AT1R-CFP/Fas-YFP

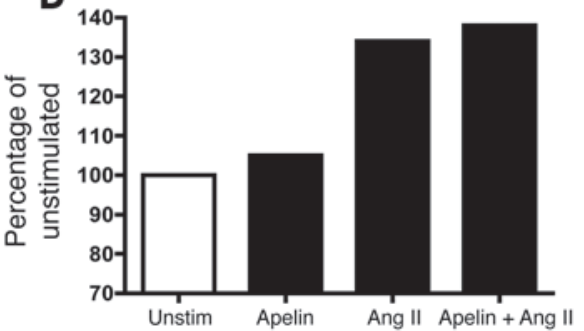

Figure 6

AT1R and APJ receptors form heterodimer complexes. (A) Immunoprecipitation using $\alpha$-GFP antibody, followed by Western blot with $\alpha-\mathrm{HA}$ antibody to detect APJ demonstrated a physical association between the 2 receptors. In the bottom panel control blot, the filled circle identifies AT1R-GFP and the triangle represents GFP. The band present in both lanes is the immunoprecipitating antibody detected by the secondary antibody. Numbers on the right reflect weights of protein standards in $\mathrm{kDa}$. The lanes are from the same gel that were noncontiguous. (B) FRET analysis was performed with HEK293 cells cotransfected with APJ and AT1R expression constructs, with coding regions fused to either CFP or YFP. Specific interaction between APJ and AT1R is shown by increased FRET signal (blue curve) but not between either of these receptors and the Fas receptor (red curve). (C) Immunoprecipitation of AT1R-GFP and blotting of APJ-HA was employed to show increased association in the presence of Ang II at 1 hour and even greater association at 6 hours. Coadministration of apelin resulted in a modest increase in association compared with Ang II alone at 6 hours. A13, apelin-13. (D) FRET analysis showed an increase in AT1R and APJ association when cells were stimulated with Ang II. Addition of apelin led to a modest increase in FRET signal in cells treated with apelin alone compared with unstimulated cells and Ang II plus apelin-treated cells compared with cells treated with Ang II alone.

the AT1R receptor, as there was no inhibition of vasopressin-stimulated nuclear signaling by vasopressin receptors through the NF- $\mathrm{KB}$ consensus reporter (Figure 7C). We also conducted a knockdown experiment to confirm the inhibitory effect of APJ expression on AT1R signaling. Using HEK293 cells, we performed knockdown experiments using siRNA against APJ to decrease the native basal APJ expression. Cells transfected with the NF- $\mathrm{\kappa B}$ luciferase reporter construct with the AT1R expression plasmid, in conjunction with either control siRNA or APJ siRNA, were stimulated with Ang II. Cells transfected with APJ siRNA demonstrated a greater response to Ang II compared with cells transfected with control siRNA, further confirming our findings that the APJ receptor inhibits AT1R signaling (3.6- versus 2.5-fold increase for $10 \mathrm{nM}$ Ang II $[P<0.05]$ and 5.4- versus 3.5-fold increase for $100 \mathrm{nM}$ Ang II $[P<0.01]$ for APJ siRNA versus control siRNA) (Figure 7D).

\section{Discussion}

Data reported here show that apelin blocks a spectrum of Ang IImediated effects on atherosclerosis in the ApoE-KO model. These endpoints result from a number of pathophysiological processes that are regulated by Ang II in the setting of vascular wall disease, including direct effects on cellular adhesion, proliferation, on the balance of reactive oxygen species. The related decrease in $\mathrm{NO}$ availability and the inflammatory consequences of increased $\mathrm{O}_{2}{ }^{-}$- signaling in vascular wall cells are thought to be intimately involved in the disease process (36). Prior studies of apelin's effects on the vascular wall have focused on the endothelium and the eNOS-dependent production of NO, where apelin potentiates phosphorylation of Akt and eNOS $(13,15,37)$. It thus seemed likely that one mechanism for apelin-mediated amelioration of atherosclerosis might be related to increased $\mathrm{NO}$ production, and indeed, experiments reported here show that apelin does increase production of NO, and that L-NAME blocks apelin-mediated amelioration of Ang II-exacerbated vascular disease in the ApoE-KO model. Increased $\mathrm{NO}$ is expected to quench $\mathrm{O}_{2}{ }^{-}$, and thus mitigate the pathological effects of $\mathrm{O}_{2}{ }^{-}$production in the vessel wall, and this is a likely mechanism for some of the in vivo effects of apelin documented in this work. In the models employed in these experiments, there was a decrease in $\mathrm{O}_{2}{ }^{-}-$that correlated with disease improvement. Evidence against this being the sole mechanism for the disease-inhibiting actions of apelin is the observation that L-NAME had no effect on $\mathrm{O}_{2}{ }^{-}$- levels in the group treated with both apelin and Ang II. It thus seems likely that additional actions of apelin are an integral part of its Ang II inhibiting functions. While 
A
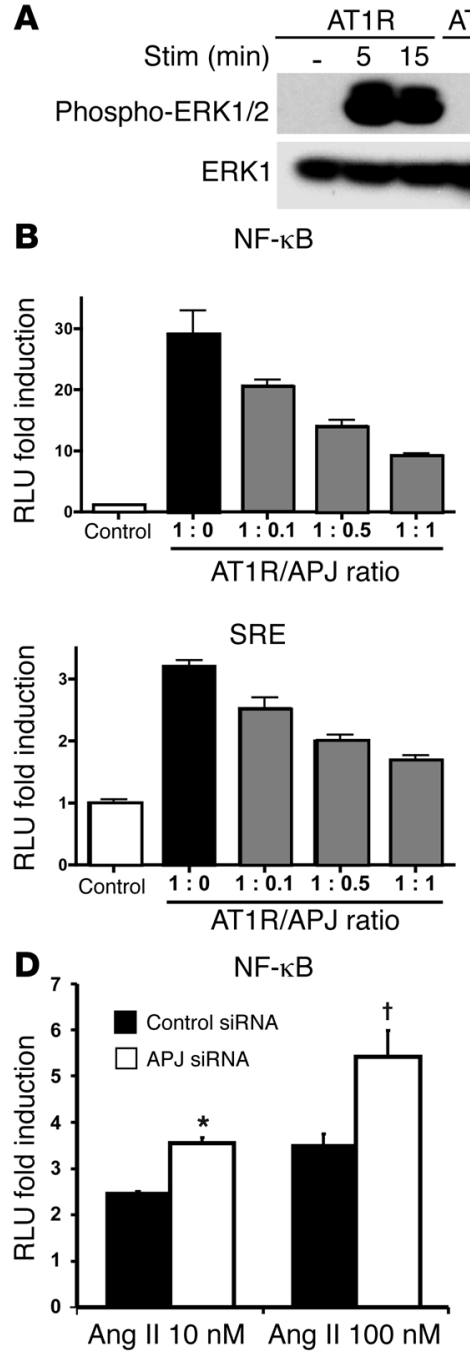

most of these data are focused on apelin blocking the actions of exogenous Ang II, our findings also support the hypothesis that apelin infusion antagonizes the actions of endogenous Ang II. We found significant increases in NO bioavailability (Figure 4A), with significant decreases in native atherosclerosis in the aortic root (Supplemental Figure 2) and $\mathrm{O}_{2}{ }^{-}$levels in aortas from $A p o E$ mice treated with apelin (Figure 4, B and C). In the face of existing data suggesting a prominent role of Ang II in mediating atherosclerosis in hypercholesterolemic mice, it is plausible that the beneficial effects of apelin, in the absence of exogenous Ang II infusion, is at least in part due to antagonism of increased levels of endogenous Ang II in ApoE-KO mice $(38,39)$.

Cellular studies reported here suggest additional mechanisms for the inhibition of Ang II exacerbation of atherosclerosis. Most significant are the data documenting a direct inhibitory effect of Ang II-mediated increases in gene transcription by concurrent cellular stimulation with apelin. These data establish for what we believe is the first time a direct antagonism at the level of cytoplasmic or nuclear signaling between these 2 peptide ligand pathways. This apelin-mediated antagonism of Ang II signaling correlates with the observed decrease in disease endpoints. These data are intriguing, given the very similar receptor structure and likely similarity between $\mathrm{G}$ protein coupling and

\section{Figure 7}

APJ receptor regulates Ang II signaling via AT1R independent of apelin. (A) Western blotting of cells transfected with AT1R alone or with APJ revealed decreased phosphorylation of ERK $1 / 2$ in cells coexpressing APJ. The bottom panel shows control blot with total ERK1 antibody. (B) NF-KB and SRE reporter constructs transfected with an $A T 1 R$ expression construct and varying amounts of APJ expression construct showed decreased signaling with greater APJ/AT1R ratios. Graphs represent mean \pm SEM. (C) Coexpression of APJ did not inhibit vasopressin-induced nuclear signaling by the vasopressin receptors (AVPR1A and AVPR2) through the NF-KB consensus reporter. (D) Luciferase analysis using an NF- $\mathrm{B}$ reporter construct with siRNA directed against APJ demonstrated enhanced Ang II response compared with control siRNA $\left({ }^{\star} P<0.05,{ }^{\dagger} P<0.01\right)$.

regulatory proteins employed. Given that a number of Ang II downstream signaling targets were blocked by apelin and that apelin did not block activation of the shared downstream targets of other stimuli such as TNF- $\alpha$ and $\mathrm{H}_{2} \mathrm{O}_{2}$, it seemed likely that crosstalk between the Ang II and apelin pathways was occurring at a proximal point in the signaling cascades.

The receptor-receptor interaction studies described here demonstrate one potential molecular mechanism of crosstalk between the apelin and Ang II pathways. Two different experimental approaches employed in these studies have shown that AT1R and APJ physically associate in the cell, presumably on the cell membrane, and this association of APJ appears to inhibit AT1R signaling in a stoichiometric fashion. Ang II-mediated downstream protein phosphorylation and reporter gene activation were both inhibited by expression of APJ in cells also expressing AT1R. We have shown that this association is dynamically regulated by Ang II levels in vitro but did not find evidence that apelin regulates this interaction to a significant degree. These experiments do not exclude the possibility that apelin can modulate receptor-receptor interaction. Further experiments are necessary to define the apelin-mediated modulation of AT1R receptor signaling, which may involve further downstream mechanisms not investigated in the present study. One additional potential mechanism is ligand-mediated variation in receptor expression levels, as relative expression level of the 2 receptors would be expected to have a significant effect on the interaction. Also, in vitro culture conditions and the phenotype of the cultured cells might not accurately reflect the situation in native cells in the diseased tissues.

If apelin does regulate receptor heterodimerization, it could account for some portion of the inhibitory signaling crosstalk that was demonstrated and modulate Ang II signaling in vivo in the disease models. There is now convincing data that such GPCR interactions can promote disease. For instance, interaction of AT1R and the bradykinin B2 receptor is a critical aspect of the preeclampsia syndrome, with AT1R-B2 heterodimerization increasing responsiveness to Ang II (40). Given the ability of APJ to inhibit AT1R signaling, one important fact that emerges from these studies is the critical importance of disease-related APJ expression level, which is worthy of careful follow-up study in these and other model systems.

While we have provided evidence for crosstalk between the apelin and Ang II signaling pathways inside the cell, it is important to consider which cells in the vessel wall might be the site of such interaction. If the observed effects in the models are due to apelin stimulation of eNOS and resulting increased $\mathrm{NO}$ production, the 
endothelial cell is obviously the cell type primarily involved. Also, in the endothelial cell, blocking Ang II signaling would decrease the well characterized proatherosclerotic effects of Ang II, such as increased adhesion molecule expression, no matter if this occurred at the receptor or intracellular signaling level. Both the APJ and AT1R are known to be expressed by the endothelium, although APJ expression in the adult vasculature has not been thoroughly characterized for either normal or diseased vessel wall. We have employed SMC in some of our experiments, because they are the only cell type that we have found to reliably express both APJ and AT1R in vitro. However, it is unclear whether APJ is expressed on these cells in vivo in atherosclerotic tissues. In fact, radioligand binding studies have shown coronary artery medial receptor expression to be low at baseline and to not be increased in atherosclerotic coronary tissue (41). There is also very little data regarding APJ expression by macrophages, with one study showing that brain-derived macrophages do not express APJ in culture (42). Further studies are necessary to elucidate the role of apelin signaling in these cell types.

While this work was being prepared for publication, Hashimoto et al. reported data suggesting conclusions contrary to those derived from studies reported here. In their studies, the ApoE-KO mice lacking APJ expression had significantly reduced aortic atherosclerosis and $\mathrm{O}_{2}{ }^{--}$production (43). This is in contrast to our findings in the infusion models as well as our studies of the apelin-ApoE double-knockout mice, in which apelin's effects were clearly antiatherosclerotic. There are a number of differences in the experimental designs of the 2 studies that likely account for the apparent inconsistency of results produced with these different experimental approaches. The genetic background employed in the animal models in the 2 sets of experiments were markedly different, and there is good evidence that modifying loci can significantly influence vascular disease mouse models $(44,45)$. The other group employed mice that were fed a very high (1\%) cholesterol diet for 15 weeks, while the mice used in our studies were fed a moderate $(0.2 \%)$ cholesterol diet for shorter durations. Also, Ang II signaling may undergo compensatory downregulation in the APJ-KO mice due to loss of apelin signaling, and this possibility needs to be investigated. Finally, it is possible that alternative genetic loci are developmentally activated in the APJ-KO animals and serve to compensate for loss of apelin-APJ signaling in the disease setting in the adult. Expression of these compensatory loci might account for the phenotype presumed to be due to loss of apelin-APJ. It will be important to repeat these experiments with an inducible recombinase system that allows the functional deletion of the apelin-APJ pathway in the adult animal, which should prevent the confounding activation of compensatory pathways with partially overlapping function, and to perform these studies on the C57BL/6 background that was used in the studies reported here.

In summary, these data establish that apelin can block a number of Ang II-related pathological processes associated with atherosclerosis, including the production of $\mathrm{O}_{2}{ }^{-}$in the vascular media. This inhibitory activity is likely mediated by increased endothelial NO production and blocking Ang II signaling to the nucleus, with some portion of the latter mediated by physical association of AT1R and APJ. These data suggest a role for the apelin-APJ pathway in atherosclerosis and Ang II-mediated vascular remodeling and provide directions for additional studies further investigating the mechanisms of the observed effects.

\section{Methods}

Animal models. Animal care was provided in accordance with the Laboratory Animal Welfare Act, the Guide for the care and use of laboratory animals (NIH publication no. 78-23. Revised 1978), and the Stanford University School of Medicine guidelines and policies for the use of laboratory animals for research and teaching, and all experimental protocols were reviewed and approved by the Administrative Panel on Laboratory Animal Care at Stanford University. Mice were maintained in a temperature-controlled $\left(20^{\circ} \mathrm{C}-22^{\circ} \mathrm{C}\right)$ environment with a 12 -hour light-dark cycle. Sterile water and standard chow diet (bypass graft model) or Western high-fat diet (abdominal atherosclerosis and aneurysm model) (21\% anhydrous milk fat and $0.15 \%$ cholesterol (Dyets no. 101511; Dyets) were available ad libitum. All mice were C57BL/6J ApoE-KO male mice (The Jackson Laboratory), between the ages of 8 and 12 weeks.

For both the aneurysm and vein graft models, a total of 6 groups of ApoE-KO mice, receiving 4-week infusions with $200 \mu$ losmotic minipumps (model 2004; Alzet Osmotic pumps), implanted subcutaneously according to the manufacturers instructions, were compared. The groups were as follows: (a) saline; (b) apelin-13 (American Peptide) $(2 \mathrm{mg} / \mathrm{kg} /$ day); (c) apelin$13(2 \mathrm{mg} / \mathrm{kg} /$ day) plus Ang II (Sigma-Aldrich) $(1.4 \mathrm{mg} / \mathrm{kg} /$ day); (d) Ang II $(1.4 \mathrm{mg} / \mathrm{kg} /$ day); (e) Ang II plus hydralazine (Sigma-Aldrich) $(250 \mathrm{mg} / \mathrm{l}$ in drinking water changed weekly); and (f) apelin plus Ang II plus L-NAME (Sigma-Aldrich) (100 mg/l in drinking water changed weekly). Apelin-13 used in these experiments was the pyroglutamated form. AAA was defined as more than or equal to $50 \%$ enlargement of the maximal abdominal aorta diameter. Animals were observed daily, and in the case of premature sudden death, necropsy was performed to determine the cause of death.

Venous bypass grafting was used as model of vascular injury for the development of neointimal hyperplasia and accelerated atherosclerosis (46). Osmotic minipumps were implanted 24 hours prior to vein graft surgery to allow adequate circulating levels of apelin/Ang II at the time of vascular injury. Vein graft surgery was performed as described previously (46). Briefly, 10- to 12-week-old male mice were anesthetized using inhaled isoflurane. The right common carotid artery was isolated and mobilized from the thoracic inlet to the bifurcation, divided at its midpoint, and cuffs were placed over the ends. The artery was inverted over the cuffs and ligated with 8-0 silk sutures. The supradiaphragmatic vena cava from an isogenic female littermate donor mouse was harvested and grafted as an interposition graft by sleeving the vein over the 2 ends of the carotid artery and ligating it with 8-0 silk sutures.

For apelin-ApoE double-knockout experiments, a previously described apelin-null allele was bred onto the C57BL/ 6 background for 10 generations, and these mice were bred with $A p o E-\mathrm{KO}$ mice on the C57BL/6 background to generate apelin-ApoE double-knockout mice (6). Male and female mice were started at 4 weeks of age on a Western diet $(0.2 \%$ cholesterol, $21.0 \%$ milk fat, $19.5 \%$ casein) or continued on normal chow diet and were maintained on these diets for 12 weeks. All mice were euthanized at the age of 16 weeks, and the aortas were isolated and processed for en face analysis.

Blood pressure and heart rate were determined noninvasively using a computerized tail-cuff system (BP 2000 Blood Pressure Analysis System; Visitech Systems Inc.) described previously (47). Mice were acclimatized to the apparatus during daily sessions over 6 days, then blood pressure measurements were obtained on a twice weekly basis during the duration of the infusion.

Tissue preparation, histology, and lesion quantification. Mice were euthanized 28 days following implantation of osmotic minipumps. The thoracic cage was removed and the animals were perfused with PBS solution for 2 minutes. The entire aorta and vein graft were exposed, and the periadventitial tissue and scar tissue were dissected and removed. Sub- 
sequently, mice were perfusion fixed in situ with $4 \%$ phosphate-buffered paraformaldehyde. In some animals, aorta and lung were removed fresh for biochemical assays.

Aorta and vein grafts were excised, fixed in paraformaldehyde overnight, dehydrated in graded ethanol solution, and paraffin embedded or removed fresh and snap frozen in OCT. For graft analysis, prior to embedding, each graft was divided at its midpoint to provide sections from the body of the graft, avoiding the cuff anastomoses. Grafts were sectioned at $150 \mu \mathrm{M}$ from the midpoint, collecting $5-\mu \mathrm{M}$ sections. Three representative sections, separated by $50 \mu \mathrm{M}$, were stained with Masson/Goldner stain for analysis. Lesion quantification was similar to that described previously (21). Vessel wall area was determined by subtracting the luminal area from total vessel area. Neointimal area was defined as the area inside the internal elastic lamina. Image analysis was performed using Image Pro Plus software (Media Cybernetics).

Aortic atherosclerosis lesion area was determined as described previously (48). Briefly, the arterial tree was perfused with PBS ( $\mathrm{pH} 7.3$ ) and then perfusion fixed with phosphate-buffered paraformaldehyde (3\%, pH 7.3). The heart and the full-length of the aorta-to-iliac bifurcation was exposed and dissected carefully from any surrounding tissues. Aortas were then opened along the ventral midline and dissected free of the animal and pinned out flat, intimal side up, onto black wax. Aortic images were captured with a digital camera mounted on a Nikon stereomicroscope and analyzed using Adobe Photoshop Extended software. The percentage of lesion area was calculated as total lesion area divided by total surface area. For mice with AAA, the area of the aneurysm was excluded from the calculations.

The atherosclerotic lesions in aortic valve area were analyzed as described previously (49). The aortic samples were perfused with PBS, fixed with paraformaldehyde (4\%), embedded in OCT, and sectioned at $10-\mu \mathrm{M}$ thickness. Five sections at $100-\mu \mathrm{M}$ intervals were collected from each mouse and stained with Masson trichrome. The lesion areas were measured and quantified using Image Pro Plus software (Media Cybernetics).

Immunohistochemistry was performed as described previously (50). Briefly, paraffin-embedded vein graft sections were stained for SMC $\alpha$-actin using a mouse monoclonal antibody (Clone A14; Sigma-Aldrich). Sections were first rehydrated in PBS, blocked for 4 hours at room temperature with horse serum (Vector Laboratories), and stained with antibody (1:100 dilution in horse serum) at $4^{\circ} \mathrm{C}$ overnight. The next day, specimens were washed in PBS, followed by Tris- $\mathrm{HCl}(20 \mathrm{mM}, \mathrm{pH}$ 8.3), and then the mixtures of alkaline phosphate-conjugated avidin-biotin complex (Vector Laboratories) and Vector Red substrate (Vector Laboratories) were added for 10 minutes. Specimens were washed in Tris- $\mathrm{HCl}$ again and then in PBS and distilled water and were mounted. Stained area was determined as described above for atherosclerotic lesion area.

Lipid analysis. Total plasma cholesterol (CHOD-PAP; Roche Diagnostics), HDL (HDL-C-plus 2nd generation; Roche Diagnostics), and triglyceride concentrations (GPO-PAP; Roche Diagnostics) were measured using enzymatic kits on an automated analyzer (Roche) according to the manufacturer's instructions.

EPR spectroscopy. EPR spectroscopy was used to quantify vascular NO production according to previously described and validated methods (51). In brief, freshly harvested lung homogenates ( $n=4-8$ per group) were stimulated with calcium ionophore (A23187; $1 \mu \mathrm{mol} / \mathrm{l})$ in $250 \mu \mathrm{l}$ KrebsHEPES buffer and then incubated with colloid $\mathrm{Fe}(\text { DETC })_{2}(285 \mu \mathrm{M})$ at $37^{\circ} \mathrm{C}$ for 90 minutes. After incubation, lung homogenates were snap frozen in a column of Krebs-HEPES buffer in liquid nitrogen, and EPR spectra were obtained using an EPR spectrometer (Model 8400; Resonance Instruments). Signals were quantified by measuring the total amplitude, after correction of baseline, and after subtracting background signals from incubation with colloid $\mathrm{Fe}(\mathrm{DETC})_{2}$ alone. Human umbilical vein endothelial cells (Lonza Inc.) served as the positive control.
Lucigenin-enhanced chemiluminescence. Total $\mathrm{O}_{2}{ }^{-}$was measured by lucigenin-enhanced chemiluminescence as described previously (52). Aortas or vein grafts were harvested, flushed with Krebs-HEPES buffer, opened longitudinally, and divided into halves. Vessels were gassed with $95 \%$ oxygen $/ 5 \%$ carbon dioxide in warmed Krebs-HEPES buffer for 30 minutes before measurements of chemiluminescence were recorded by a luminometer (Bio Orbit) using $5 \mu \mathrm{mol} / 1$ lucigenin. One half of each vessel was incubated in L-NAME $(1 \mathrm{mmol} / \mathrm{l})$ (Sigma-Aldrich). After measuring baseline readings for 4 minutes, samples were equilibrated and dark adapted for 5 minutes, and chemiluminescence was recorded for 10 minutes. Recordings were performed blind to the sample identity. Results were expressed as counts per second per milligram of tissue dry weight.

Oxidative fluorescent microtopography. $\mathrm{O}_{2}{ }^{-{ }^{-}}$was detected in the layers of the vessel wall using fluorescent probe dihydroethidium as described previously (52). Fresh segments of upper descending thoracic aorta were frozen in OCT compound. Cryosections $(30 \mu \mathrm{m})$ were incubated with Krebs-HEPES buffer for 30 minutes at $37^{\circ} \mathrm{C}$, with or without $1 \mathrm{mmol} / 1$ L-NAME, $50 \mu \mathrm{mol} / 1$ apocynin, $50 \mathrm{U} / \mathrm{ml} \mathrm{O}_{2}{ }^{--}$dismutase (all SigmaAldrich) followed by 5 minutes dark incubation with $2 \mu \mathrm{mol} / 1$ dihydroethidium (DHE; Molecular Probes). Images were obtained on a confocal microscope (Bio-Rad MRC-1024 laser; filter settings, excitation filter at $488 \mathrm{~nm}$; emission filter at $550 \mathrm{~nm}$ ). High-power (original magnification, $\times 60$ ) images were used to measure 2-hydroxyethidium fluorescence. Endothelial fluorescence was quantified (intensity $\mathrm{x}$ area) on the luminal side of the internal elastic lamina for media between the internal and external elastic lamina and for adventitia outside the external elastic lamina. Quantification was performed using Image Pro Plus software (Media Cybernetics). Analysis was performed blind to the sample identity. Mean fluorescence was calculated from 4 separate, high-power fields from each quadrant to produce $n=1$.

Cell culture assays. For transfection experiments, HA-tagged APJ, AT1R, and arginine vasopressin receptor (AVPR1A and AVPR2) constructs in pcDNA3.1 were purchased from cDNA.org (Missouri University of Science and Technology cDNA Resource Center). A full-length AT1R cDNA was obtained from Marc Caron, Duke University, Durham, North Carolina, USA, and inserted into a pEGFP-N1 vector (Clontech). The NF-кB, AP-I, NFAT, and SRE luciferase reporter constructs were obtained from BD Biosciences. HEK293 cells were cultured in Dulbecco modified Eagle medium with $10 \%$ fetal bovine serum, 10 units $/ \mathrm{ml}$ penicillin, and $100 \mu \mathrm{g} / \mathrm{ml}$ streptomycin. Transient transfection of the HEK293 cells was carried out using FuGene reagent per manufacturer's protocols in 6-well plates. Human pulmonary artery SMCs were obtained from PromoCell and grown in growth medium per manufacturer's protocols. For ERK phosphorylation, transfected HEK293 cells were serum starved for 6 hours, incubated with the agonist for designated times at $37^{\circ} \mathrm{C}$, and immediately lysed using diluted 10X Cell Lysis Buffer (Cell Signaling Technology Inc.). For luciferase assays, transfected cells were serum starved for 6 hours and then stimulated with the designated ligand for 24 hours prior to harvest. A renilla luciferase construct was cotransfected to control for transfection efficiency in each of these experiments. For siRNA-knockdown experiments, HEK293 cells were cotransfected with either control siRNA or APJ-specific siRNA (Dharmacon). After 48 hours, cells were stimulated with varying concentrations of Ang II for 8 hours. Cells were subsequently harvested and lysates obtained. Luciferase assay was performed as per manufacturers instructions (Promega), and samples were done in triplicates.

RASMCs were harvested by enzymatic digestion from rat aortas as previously described (53). Cells were cultured in Dulbecco modified Eagle medium with $10 \%$ fetal bovine serum, 10 units $/ \mathrm{ml}$ penicillin, and $100 \mu \mathrm{g} / \mathrm{ml}$ streptomycin. After 24 hours serum starvation, the cells were stimulated 
with $100 \mathrm{nM}$ Ang II with or without $100 \mathrm{nM}$ apelin-13 and immediately lysed and frozen for protein electrophoresis.

Immunoprecipitation and immunoblotting. Immunoprecipitations were performed on cells lysed with cell lysis buffer. Lysates were precleared with protein $\mathrm{G}$ agarose, and immunoprecipitation was performed with the respective antibodies at a concentration of $1 \mu \mathrm{g} / \mathrm{ml}$ for 24 hours at $4^{\circ} \mathrm{C}$. Protein $\mathrm{G}$ agarose was added and incubated for an additional 2 hours. Western blotting of SDS-PAGE gels was performed with standard methodology, and the bands were visualized using an enhanced chemiluminescence system. $\alpha$-GFP and $\alpha$-HA antibodies were purchased from Roche.

For RASMC stimulation experiments, cells were lysed, resolved by SDSPAGE, and blotted with phospho-ERK or total ERK1 antibody (Cell Signaling Technology Inc.) followed by anti-rabbit horseradish peroxidase-conjugated secondary antibody (Sigma-Aldrich).

FRET analysis. FRET analysis was performed as previously described (www.stke.org/cgi/content/full/OC_sigtrans;2000/38/pl1) (54, 55). In brief, CFP- and YFP-tagged AT1R and APJ constructs were generated. The appropriate CFP and YFP fusion constructs were transfected into $293 \mathrm{~T}$ cells in 6-well plates. Cells were analyzed by flow cytometry 36 hours after transfection, after being resuspended by vigorous pipetting in medium. A BD LSR II System flow cytometer was used with CFP excitation at $405 \mathrm{~nm}$ and YFP excitation at $488 \mathrm{~nm}$. Detection was performed at $470 \mathrm{~nm}$ for CFP and $550 \mathrm{~nm}$ for YFP. FRET detection was performed at $550 \mathrm{~nm} / 50 \mathrm{~nm}$ bandpass using $405 \mathrm{~nm}$ excitation.

1. O’Dowd, B.F., et al. 1993. A human gene that shows identity with the gene encoding the angiotensin receptor is located on chromosome 11. Gene. 136:355-360.

2. Tatemoto, K., et al. 1998. Isolation and characterization of a novel endogenous peptide ligand for the human APJ receptor. Biochem. Biophys. Res. Commun. 251:471-476.

3. Chen, M.M., et al. 2003. A novel role for the potent endogenous inotrope apelin in human cardiac dysfunction. Circulation. 108:1432-1439.

4. Kleinz, M.J., and Davenport, A.P. 2004. Immunocytochemical localization of the endogenous vasoactive peptide apelin to human vascular and endocardial endothelial cells. Regul. Pept. 118:119-125.

5. Kleinz, M.J., Skepper, J.N., and Davenport, A.P. 2005. Immunocytochemical localisation of the apelin receptor, APJ, to human cardiomyocytes, vascular smooth muscle and endothelial cells. Regul. Pept. 126:233-240.

6. Sheikh, A.Y., et al. 2007. In vivo genetic profiling and cellular localization of apelin reveals a hypoxiasensitive, endothelial-centered pathway activated in ischemic heart failure. Am. J. Physiol. Heart Circ. Physiol. 294:H88-H98.

7. Ashley, E.A., et al. 2005. The endogenous peptide apelin potently improves cardiac contractility and reduces cardiac loading in vivo. Cardiovasc. Res. 65:73-82.

8. Atluri, P., et al. 2007. Ischemic heart failure enhances endogenous myocardial apelin and APJ receptor expression. Cell. Mol. Biol. Lett. 12:127-138.

9. Farkasfalvi, K., et al. 2007. Direct effects of apelin on cardiomyocyte contractility and electrophysiology. Biochem. Biophys. Res. Commun. 357:889-895.

10. Szokodi, I., et al. 2002. Apelin, the novel endogenous ligand of the orphan receptor APJ, regulates cardiac contractility. Circ. Res. 91:434-440.

11. Jia, Y.X., et al. 2006. Apelin protects myocardial injury induced by isoproterenol in rats. Regul. Pept. 133:147-154.

12. Cheng, X., Cheng, X.S., and Pang, C.C. 2003. Venous dilator effect of apelin, an endogenous peptide ligand for the orphan APJ receptor, in conscious rats. Eur. J. Pharmacol. 470:171-175.
Statistics. Data are presented as mean \pm SEM. Data were subjected to the Kolmogorov-Smirnov test to determine distribution. Groups were compared using the Mann-Whitney $U$ test for nonparametric data or the Student's $t$ test for parametric data. When comparing multiple groups, data were analyzed by ANOVA with Bonferroni's post test. For multiple testing for parametric data, a value of $P<0.05$ was considered statistically significant.

\section{Acknowledgments}

This work was supported in part by NIH RO1 grant HL077676 (to T. Quertermous), Stanford School of Medicine Dean's Fellowship (to H.J. Chun), and Division of Intramural Research, National Institute of Allergy and Infectious Diseases, NIH (to M.J. Lenardo and L. Zheng).

Received for publication December 26, 2007, and accepted in revised form July 16, 2008.

Address correspondence to: Thomas Quertermous, Division of Cardiovascular Medicine, Stanford University, 300 Pasteur Drive, Stanford, California 94305, USA. Phone: (650) 723-5013; Fax: (650) 725-2178; E-mail: tomq1@stanford.edu.

Hyung J. Chun, Ziad A. Ali, and Yoko Kojima contributed equally to this work.
13. Ishida, J., et al. 2004. Regulatory roles for APJ, a seventransmembrane receptor related to AT1, in blood pressure in vivo. J. Biol. Chem. 279:26274-26279.

14. Lee, D.K., et al. 2000. Characterization of apelin, the ligand for the APJ receptor. J. Neurochem. 74:34-41.

15. Tatemoto, K., et al. 2001. The novel peptide apelin lowers blood pressure via a nitric oxide-dependent mechanism. Regul. Pept. 99:87-92.

16. O'Carroll, A.M., Don, A.L., and Lolait, S.J. 2003. APJ receptor mRNA expression in the rat hypothalamic paraventricular nucleus: regulation by stress and glucocorticoids. J. Neuroendocrinol. 15:1095-1101.

17. De Mota, N., et al. 2004. Apelin, a potent diuretic neuropeptide counteracting vasopressin actions through inhibition of vasopressin neuron activity and vasopressin release. Proc. Natl. Acad. Sci. U. S. A. 101:10464-10469.

18. Lee, D.K., George, S.R., and O'Dowd, B.F. 2006. Unravelling the roles of the apelin system: prospective therapeutic applications in heart failure and obesity. Trends Pharmacol. Sci. 27:190-194.

19. Iwanaga, Y., Kihara, Y., Takenaka, H., and Kita, T. 2006. Down-regulation of cardiac apelin system in hypertrophied and failing hearts: Possible role of angiotensin II-angiotensin type 1 receptor system. J. Mol. Cell. Cardiol. 41:798-806.

20. Daugherty, A., Manning, M.W., and Cassis, L.A. 2000. Angiotensin II promotes atherosclerotic lesions and aneurysms in apolipoprotein E-deficient mice. J. Clin. Invest. 105:1605-1612.

21. Dietrich, H., et al. 2000. Rapid development of vein graft atheroma in ApoE-deficient mice. Am. J. Pathol. 157:659-669.

22. Piedrahita, J.A., Zhang, S.H., Hagaman, J.R., Oliver, P.M., and Maeda, N. 1992. Generation of mice carrying a mutant apolipoprotein $\mathrm{E}$ gene inactivated by gene targeting in embryonic stem cells. Proc. Natl. Acad. Sci. U. S. A. 89:4471-4475.

23. Zhang, S.H., Reddick, R.L., Piedrahita, J.A., and Maeda, N. 1992. Spontaneous hypercholesterolemia and arterial lesions in mice lacking apolipoprotein E. Science. 258:468-471.

24. Xu, Q., Zhang, Z., Davison, F., and Hu, Y. 2003. Circulating progenitor cells regenerate endothelium of vein graft atherosclerosis, which is diminished in ApoE-deficient mice. Circ. Res. 93:e76-e86.
25. Abbasi, S., Su, B., Kellems, R.E., Yang, J., and Xia, Y. 2005. The essential role of MEKK3 signaling in angiotensin II-induced calcineurin/nuclear factor of activated T-cells activation. J. Biol. Chem. 280:36737-36746.

26. Han, Y., Runge, M.S., and Brasier, A.R. 1999. Angiotensin II induces interleukin-6 transcription in vascular smooth muscle cells through pleiotropic activation of nuclear factor-kappa B transcription factors. Circ. Res. 84:695-703.

27. Lu, D., Yang, H., and Raizada, M.K. 1996. Angiotensin II regulation of neuromodulation: downstream signaling mechanism from activation of mitogenactivated protein kinase. J. Cell Biol. 135:1609-1617.

28. AbdAlla, S., Lother, H., Abdel-tawab, A.M., and Quitterer, U. 2001. The angiotensin II AT2 receptor is an AT1 receptor antagonist. J. Biol. Chem. 276:39721-39726.

29. AbdAlla, S., Lother, H., and Quitterer, U. 2000. AT1-receptor heterodimers show enhanced G-protein activation and altered receptor sequestration. Nature. 407:94-98.

30. Minneman, K.P. 2007. Heterodimerization and surface localization of $\mathrm{G}$ protein coupled receptors. Biochem. Pharmacol. 73:1043-1050.

31. Bafford, R., Sui, X.X., Wang, G., and Conte, M. 2006. Angiotensin II and tumor necrosis factoralpha upregulate survivin and Kruppel-like factor 5 in smooth muscle cells: Potential relevance to vein graft hyperplasia. Surgery. 140:289-296.

32. Gavazzi, G., et al. 2007. NOX1 deficiency protects from aortic dissection in response to angiotensin II. Hypertension. 50:189-196.

33. Miller, F.J., Jr., et al. 2002. Oxidative stress in human abdominal aortic aneurysms: a potential mediator of aneurysmal remodeling. Arterioscler. Thromb. Vasc. Biol. 22:560-565.

34. Tham, D.M., et al. 2002. Angiotensin II is associated with activation of NF-kappaB-mediated genes and downregulation of PPARs. Physiol. Genomics. 11:21-30.

35. Schmieder, R.E., Hilgers, K.F., Schlaich, M.P., and Schmidt, B.M. 2007. Renin-angiotensin system and cardiovascular risk. Lancet. 369:1208-1219.

36. Mehta, P.K., and Griendling, K.K. 2006. Angiotensin II cell signaling: physiological and pathological 
effects in the cardiovascular system. Am. J. Physiol. Cell Physiol. 292:C82-C97.

37. Zhong, J.C., et al. 2007. Apelin modulates aortic vascular tone via endothelial nitric oxide synthase phosphorylation pathway in diabetic mice. Cardiovasc. Res. 74:388-395.

38. Daugherty, A., Rateri, D.L., Lu, H., Inagami, T., and Cassis, L.A. 2004. Hypercholesterolemia stimulates angiotensin peptide synthesis and contributes to atherosclerosis through the AT1A receptor. Circulation. 110:3849-3857.

39. Hayek, T., Attias, J., Smith, J., Breslow, J.L., and Keidar, S. 1998. Antiatherosclerotic and antioxidative effects of captopril in apolipoprotein E-deficient mice. J. Cardiovasc. Pharmacol. 31:540-544.

40. AbdAlla, S., Lother, H., el Massiery, A., and Quitterer, U. 2001. Increased AT(1) receptor heterodimers in preeclampsia mediate enhanced angiotensin II responsiveness. Nat. Med. 7:1003-1009.

41. Katugampola, S.D., Kuc, R.E., Maguire, J.J., and Davenport, A.P. 2002. G-protein-coupled receptors in human atherosclerosis: comparison of vasoconstrictors (endothelin and thromboxane) with recently de-orphanized (urotensin-II, apelin and ghrelin) receptors. Clin. Sci. (Lond.) 103(Suppl.
48):171S-175S

42. Choe, W., et al. 2000. Functional expression of the seven-transmembrane HIV-1 co-receptor APJ in neural cells. J. Neurovirol. 6(Suppl. 1):S61-S69.

43. Hashimoto, T., et al. 2007. Requirement of apelinapelin receptor system for oxidative stress-linked atherosclerosis. Am. J. Pathol. 171:1705-1712.

44. Harmon, K.J., Couper, L.L., and Lindner, V. 2000. Strain-dependent vascular remodeling phenotypes in inbred mice. Am. J. Pathol. 156:1741-1748.

45. Knowles, J.W., and Maeda, N. 2000. Genetic modifiers of atherosclerosis in mice. Arterioscler. Thromb. Vasc. Biol. 20:2336-2345.

46. Zou, Y., Dietrich, H., Hu, Y., Metzler, B., Wick, G., and Xu, Q. 1998. Mouse model of venous bypass graft arteriosclerosis. Am. J. Pathol. 153:1301-1310.

47. Johns, C., Gavras, I., Handy, D.E., Salomao, A., and Gavras, H. 1996. Models of experimental hypertension in mice. Hypertension. 28:1064-1069.

48. Tabibiazar, R., et al. 2005. Mouse strain-specific differences in vascular wall gene expression and their relationship to vascular disease. Arterioscler. Thromb. Vasc. Biol. 25:302-308.

49. Paigen, B., Morrow, A., Holmes, P.A., Mitchell, D., and Williams, R.A. 1987. Quantitative assessment of atherosclerotic lesions in mice. Atherosclerosis. 68:231-240.

50. Ali, Z.A., et al. 2005. Gene transfer of a broad spectrum CC-chemokine inhibitor reduces vein graft atherosclerosis in apolipoprotein E-knockout mice. Circulation. 112:I235-I241.

51. Kleschyov, A.L., and Munzel, T. 2002. Advanced spin trapping of vascular nitric oxide using colloid iron diethyldithiocarbamate. Methods Enzymol. 359:42-51.

52. Alp, N.J., et al. 2003. Tetrahydrobiopterin-dependent preservation of nitric oxide-mediated endothelial function in diabetes by targeted transgenic GTP-cyclohydrolase I overexpression. J. Clin. Invest. 112:725-735.

53. Sista, A.K., et al. 2005. Increased aortic stiffness in the insulin-resistant Zucker fa/fa rat. Am. J. Physiol. Heart Circ. Physiol. 289:H845-H851.

54. Chan, F.K., et al. 2000. A domain in TNF receptors that mediates ligand-independent receptor assembly and signaling. Science. 288:2351-2354.

55. Siegel, R.M., et al. 2000. Measurement of molecular interactions in living cells by fluorescence resonance energy transfer between variants of the green fluorescent protein. Sci. STKE. 2000:PL1. 